\title{
Conf- $920930-1$
}

\section{Fermi National Accelerator Laboratory}

Prons

MAR 02

FNAL/C- $-92 / 13$

DE9 2008249

\section{High Performance Parallel Local Memory Computing at Fermilab}

\author{
T. Nash \\ Computing Division \\ Fermi National Accelerator Laboratory \\ P.O. Box 500, Batavia, Illinois 60510
}

January 1992

Published in the Proceedings of WHP92 on Heterogeneous Processing, Beverly Hills, California, March 23, 1992.

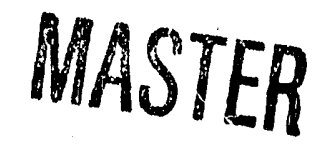




\section{Disclaimer}

This report was prepared as an account of work sponsored by an agency of the United States Government. Neither the United States Government nor any agency thereof, nor any of their employees, makes any warranty, express or implied, or assumes any legal liability or responsibility for the accuracy, completeness, or usefulness of any information, apparatus, product, or process disclosed, or represents that its use would not infringe privately owned rights. Reference herein to any specific commercial product, process, or service by trade name, trademark, manufacturer, or otherwise, does not necessarily constitute or imply its endorsement, recommendation, or favoring by the United States Government or any agency thereof. The views and opinions of authors expressed herein do not necessarily state or reflect those of the United States Government or any agency thereof. 


\title{
High Performance Parallel Local Memory Computing at Fermilab
}

\author{
Presented by Thomas Nash* \\ Computing Division \\ Fermi National Accelerator Laboratory, Batavia IL 60510
}

\section{Introduction}

Both experimental and theoretical approaches to high energy physics (HEP) at Fermilab are computer technology limited: no one can identify a requirement on computing or data capacity that is independent of cost or other realities. Fermilab has been forced, therefore, to turn significant attention and resources to finding extremely cost effective solutions to its computing using whatever technology is available, and in the process has become a pioneer in goal-driven computer science, integrating commercial solutions at the chip, board, and system level

The most effective HEP computing solutions are general purpose parallel systems which take advantage of the scientist's ability and willingness to identify explicitly the structure of the problem. Science, almost by definition, deals with regular problems which intrinsically offer parallel solution. It is straightforward to recognize that a lattice problem maps obviously to a grid of processors. Similarly, it is almost intuitive to recognize that the independent events resulting from the collision of particles in a HEP experiment can be dealt, one at a time, to the individual nodes of an event oriented parallel farm of computers. This explicit parallelism is the disting-uishing feature of the advances in computing developed in the HEP context. In recent years, most obviously first in the hypercube movement, and now in the surge of interest in networked parallel workstation "clusters", explicit parallelism has become a recognized force in the computer science attack on the problems of parallel computing.

Early work on parallel processing led directly to the two main explicitly parallel, local memory computing directions that dominate Fermilab's high performance computing today: farms of RISC technology workstations for experiment triggering and reconstruction, and the ACPMAPS massively parallel computer that supports theoretical calculations. Likewise, early efforts in software tools which allow the scientist to focus on the problem not the architecture

\footnotetext{
* This report summarizes the efforts of many individuals in the Computing Division at Fermilab. Thanks to E. Schermerhorn, M. Fausey, M. Fischler, R. Pordes, S. Wolbers, for preparing this report.
}

have evolved into the modern packages CPS and Canopy which, respectively, are used in the contexts of networked farm systems and closely coupled massively parallel systems.

\section{RISC processor farms and CPS}

Today there are over 400 nodes in farms at Fermilab. A farm is a collection of loosely coupled processors that are managed as a single system. The most recent Fermilab farms used in experiment event reconstruction consist of two commercial species of RISC workstation processor nodes: Silicon Graphics, Inc. (SGI) and IBM RS/6000.

\section{The processor farms}

The processors in a farm are connected over a communications network so they are able to exchange data. That can be VME, Ethernet, or whatever is practical. Most activity is now via TCP/IP. This allows all the computers to share data from a single set of tape and disk drives. The sharing of these peripheral devices and the fact that a farm works collectively on a single problem at a time distinguishes farms from mere collections of workstations.

Each farm is composed of approximately 100 processors, serving as worker nodes, tape server nodes, and as NFS server nodes. Each runs its vended version of a UNIX operating system. The CPUs are rack mounted in tiers and each has at least $12 \mathrm{MB}$ memory, $160 \mathrm{MB}$ disk and Ethernet capability. See Table for other information. The farms are configured into "farmlets" of approximately $12-25$ processors, and this subunit is on one Ethernet segment, separated by an Ethernet bridge or router. Each "farmlet" contains an NFS server, a tape server, and the balance are used as worker nodes. Local disk on each node has a copy of the operating system and paging space. The worker nodes all remote mount the Fermilab local executables from their "farmlet" NFS server, as well as scratch space areas for the batch jobs. Communication outside of the "farmlet" is generally limited to user logins and connections to the CPS Job Manager, which oversees the job. 


\section{Farms at Fermilab}

IBM RS/6000

SGI, Inc.

\author{
Worker nodes
}

63 Model 320s

36 Model 320Hs

22 Model 4D25s

78 Model 4D35s

3 Model 4D25s

3 Model 530

Server nodes

2 Model 320s

3 Model 4D310s

AIX 3.1.5

Operating System

IRIX 3.3.2

IRIX 3.3.3

\section{The software support}

The software environment is layered. This approach utilizes the features of the UNIX operating system, and each farm implements its own vendor's version of UNIX. Lying above that is the communications and networking layer. This contains TCP/IP and NFS at a minimum. Topmost is special software which has been written at Fermilab to make it possible for scientists to use a farm effectively in a manner similar to that of a single large computer. Called "Cooperative Processes Software" (CPS), this software runs on a variety of computer platforms, and is designed to accommodate heterogeneous farms made up of different types of computers.

CPS is a tools package which eases splitting a job across a set of processes. The processes may be distributed over a set of computers. From the standpoint of hardware the CPS environment is a set of networked UNIX workstations. However, this is not the way the scientist sees the CPS environment; the process oriented applications software environment the user encounters is a fully-connected group of processes, each having equal access to each other and peripheral devices. CPS supports general process interactions including:

data transfers; send data to another process, and get data from another process.

remote subroutine calls; a process calls a subroutine on another process.

process queues and synchronization mechanisms; determine process availability for data or remote call, and coordination of transfers and calls.

An attractive feature of CPS for many is that it can be used with or without explicit message passing. While message passing can be specified for interprocess communication, CPS organizes process execution by queuing and dequeuing from, for example, READY and DONE queues. The user may define his own set of queues. Message passing underlies this scheme, as well as the CPS subroutine calls, but it remains hidden from the user unless it is explicitly used.

\section{Important CPS subroutines}

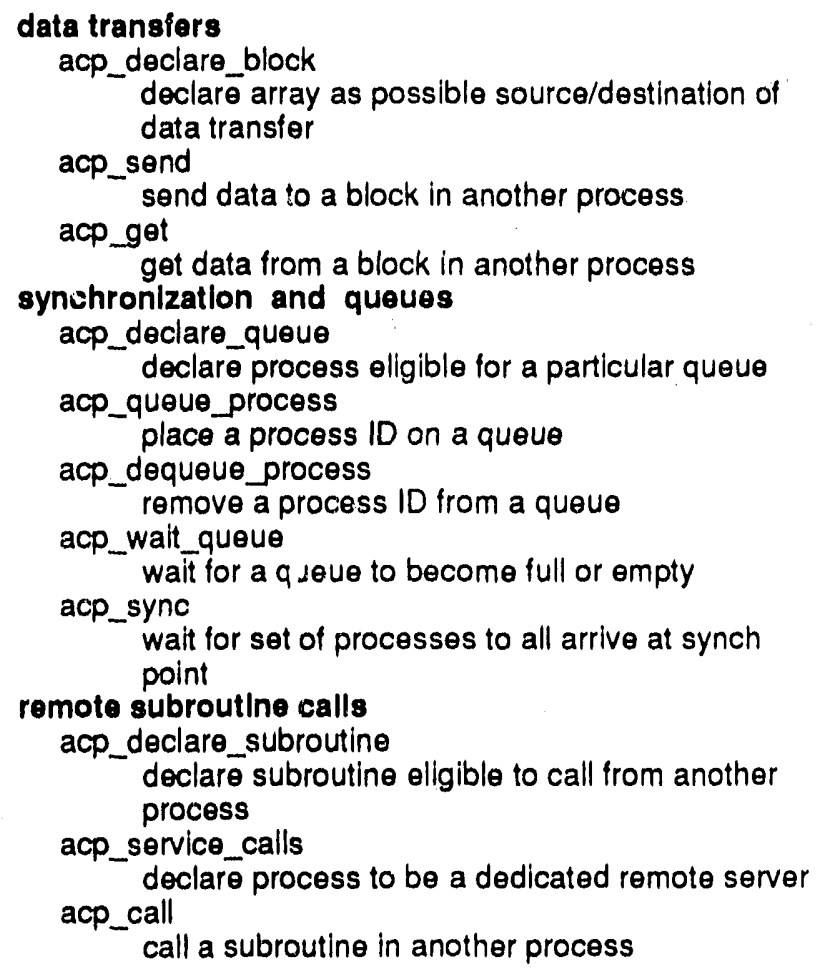

Program development with CPS begins by incorporating CPS subroutines into the application code using the tool kit of explicit parallelism directives One then compiles the program on target computer(s) and links the program on the target computer(s) with the CPS system library. Applications debugging is simplified because jobs operate identically under CPS on single or multiple computers. The job manager starts the set of processes running, manages the interactions between the processes, and shuts down the processes at the end of the job.

CPS has a batch facility that supports production jobs. Users submit CPS jobs to UNIX batch queues, one queue for each processor farm or production system, depending on how the cumplex is configured. The next job on the queue is started when a farm becomes free. The various phases in the Batch sequence - ondeck; copy; execution; and, copyback - are pipelined, allowing multiple jobs to be handled. CPS batch supports computer center operations with an operator console that displays tape mount requests and job status information.

To date, the CPS software system has been ported to a large number of hardware platforms. Current implementations at FNAL assume all processes from a job are executing on the same computer platform, running identical environments. However, the CPS primitives 
were originally designed to be platform independent, and thus offer the opportunity to manage distributed heterogeneous parallel processing. The current implementation of CPS (V2.5) can handle heterogeneous farms as has been demonstrated at a sister laboratory at another site.

We are planning a project to integrate CPS with a second Fermilab software system, UNIX Product Support (UPS) to provide a consistent automatically uniform environment across the varied platforms of heterogeneous networked system.

\section{Creating consistent environments}

As it exists now, UPS manages software packages across a set of different computer platforms, but has no functionality for job execution. Its appeal is that it simplifies and organizes the task of using a variety of computing platforms to work on the same application. It handles the housekeeping associated with developing and using applications, especially over long periods of time, in multiple computing environments.

UPS utilities provide uniform mechanisms for defining the versions of software to be used; user access to the required environmental variables and other parameters needed in order to use the software; distribution of selected software to remote computers; generation of the final application (files such as executables, data files etc associated with the job to be run); provision of a platform independent process operating environment; census of the software installed on remote computers; archival and retrieval of old versions of software; etc.

The UPS kernel supports multiple simple databases of lists of software programs with associated attributes of version, platform type, proprietary nature, and dependency of the software on other software packages. A single UPS data base can be used by multiple, different computers, or multiple data bases can be used on a single computer. UPS provides utilities for handling the information contained in these databases, and for managing the software so described.

The extensions to CPS/UPS we plan would provide the framework to support the development, building, and execution of a multiprocess application where the functions to be performed are distributed among several different platforms of UNIX computers (IBM RS/6000, SGI, DEC5000 and Sparcstation IIs) and specified portions of the data to be processed are transferred between the processes.

To support execution of the processes as a single job, the system must provide for distribution of the correct versions of the requisite files to the various computers; for the transparent transfer of data between platforms that may have different dataword byte ordering or floating point representations; for the coordinated startup, monitoring and stopping of the processes in a uniform manner across the different computer platforms; and lor a uniform and centralized mechanism for the reporting of results at the application level. All the interprocess communication, synchronization, management and data transfer mechanisms must be computer hardware and software independent.

For example, in the domain of HEP, the system we propose would allow experiment event data to be read from I/O media on a Silicon Graphics, the core of the data processed on an IBM RS/6000, and the results of the physics analysis visualized on a Decstation 5000. To achieve this requires a system that allows the application developer to specify what source files are to be compiled and linked on which platform; what dependencies these compilations and links have on each other; and what data and configuration files are needed on which platform. The system will provide for the requisite files and their updated versions to be available on the specified computer at the appropriate time. It will maintain a history of the application generation for later checking as needed by the application user or maintainer.

\section{ACPMAPS: Massively parallel computer and Canopy}

Fermilab efforts to support theoretical computations in HEP led to the development of a massively parallel local memory computer that is ideally suited for the lattice gauge calculations of quantum chromodynamics, $Q C D$, one of the biggest consumers of computer cycles nationwide. This highly parallel, cost effective computer is called the Advanced Computer Program Multi-Array Processor System, or ACPMAPS.

ACPMAPS is a multiprocessor computer with hundreds of individual processor modules that can work in parallel on a single problem. It uses an innovative scheme for communication between its constituent processors. The connectivity can be thought of as similar to a modern telephone switching network, but at much higher speeds. Each processor module resides in one of the sixteen slots of a Bus Switch Backplane (BSB) crossbar switch crate which takes on a role analogous to a local telephone exchange. The BSB allows a processor in one BSB slot to make a point-to-point connection with a processor in any other BSB slot. Once a connection has been established, the processor that initiated the connection can read and write the memory of the other processor at a rate of 20 megabytes per second. The time to establish a connection is only a few micro-seconds.

Up to eight pairs of processors may communicate simultaneously, thus providing an aggregate communication of 160 megabytes per second within the crate. An additional module, the Bus Switch Interface Board (BSIB), provides a mechanism for communicating between BSBs. A BSIB in one crate can be connected via twisted-pair cables to a BSIB in another crate. This provides a 20 megabyte per second link between the two BSB slots like a long distance call. The ACPMAPS computer makes extensive use of BSIB modules-. approximately one half of all BSB slots are filled with 
BSIBs. This patented switch hardware allows systems to be interconnected in a large variety of ways with specific configurations optimized for the type of calculation to be performed. In the Fermilab installation, targeted at lattice gauge, the system is set up as 4 fully connected $3 \times 3$ planes of crossbar crates.

ACPMAPS also contains a highly parallel $1 / 0$ subsystem. Approximately one half of the BSBs have one of their slots connected to a VME backplane in the same physical crate which in turn provides a connection to a SCSI bus. Each SCSI bus contains two 676 megabyte disk drives and two Exabyte $8 \mathrm{~mm}$ tape drives (up to two gigabytes of storage per tape).

\section{ACPMAPS upgrade}

A new ACPMAPS processor module containing two $40 \mathrm{MHz}$ Intel 860 microprocessors.per board, has been designed and built to upgrade the ACPMAPS processing power. This processor is compatible with the same BSB/BSIB communications mechanism. The original processing modules are being removed and replaced by the new modules. The commitment not to interrupt ongoing production physics dictates a cautious pace for this replacement process. Existing physics applications programs (that had already been written for ACPMAPS) run on the new modules with no changes. The underlying C software required only minor adaptations for the new processor, and a library of optimized computational kernels is being ported to the 1860 chip architecture. These software changes represent a moderate systems effort, and are invisible to the ACPMAPS user community. The upgraded ACPMAPS will have ten times the computing power of the original ACPMAPS-the ability to perform at a peak rate of 50 GFLOPS.

\section{Summary of upgrade}

\section{Existing ACPMAPS Upgraded ACPMAPS}

\section{Aggregate Performance}

\author{
5 GFLOPS (peak) \\ 2,500 Mbytes memory \\ 22,000 Mbytes disk space \\ 64,000 Mbytes of tape \\ drive capacity
}

\author{
50 GFLOPS (peak) \\ 20,000 Mbytes memory \\ 22,000 Mbytes disk space \\ 64,000 Mbytes of tape \\ drive capacity
}

\section{Constituent Parts}

\section{Canopy}

A powerful software environment is an important and innovative part of the ACPMAPS system; it is called Canopy. Canopy is designed to support computing for grid oriented problems that map onto a lattice, defined generally as a set of sites, each of which has defined directions to a set of neighboring sites.

For example, a three dimensional grid is a lattice where the set of sites is the set of all points where the grid lines cross. The calculations performed by Fermilab and collaborating physicists make use of a four-dimensional space-time lattice to perform QCD calculations of the strong force. When running lattice gauge software under Canopy, ACPMAPS typically performs at the high level of about $30 \%$ of its peak GFLOPS rating. User programming is in $\mathrm{C}$, with certain Canopy kernels and common computational kernels in assembler. The level of aistraction in Canopy remains high. The scientist uses subıoutines to create a grid with known connectivity and to manipulate the fields that are situated on these grids. Then, by writing task routines to do operations on a site, the parallelism is automatically invoked. The user need not be aware of the specific machine details such as the number of nodes it has or how the sites are distributed among the nodes.

\section{Summary}

Motivated by an ongoing need to have cost effective computing, Fermilab has made some important contributions to distributed memory parallel computing in both hardware and software. By adopting the philosophy of incorporating technology at the highest commercially available level, these products will become even more robust.
306 processors

(two i860s each)

36 BSB crates

271 BSIBs

32 disk drives

32 tape drives

$\begin{array}{ll}\text { (Weitek XL8032) } & \text { (two i860s each) } \\ 36 \text { BSB crates } & 36 \text { BSB crates } \\ 271 \text { BSIBs } & 271 \text { BSIBs } \\ 32 \text { disk drives } & 32 \text { disk drives } \\ 32 \text { tape drives } & 32 \text { tape drives }\end{array}$



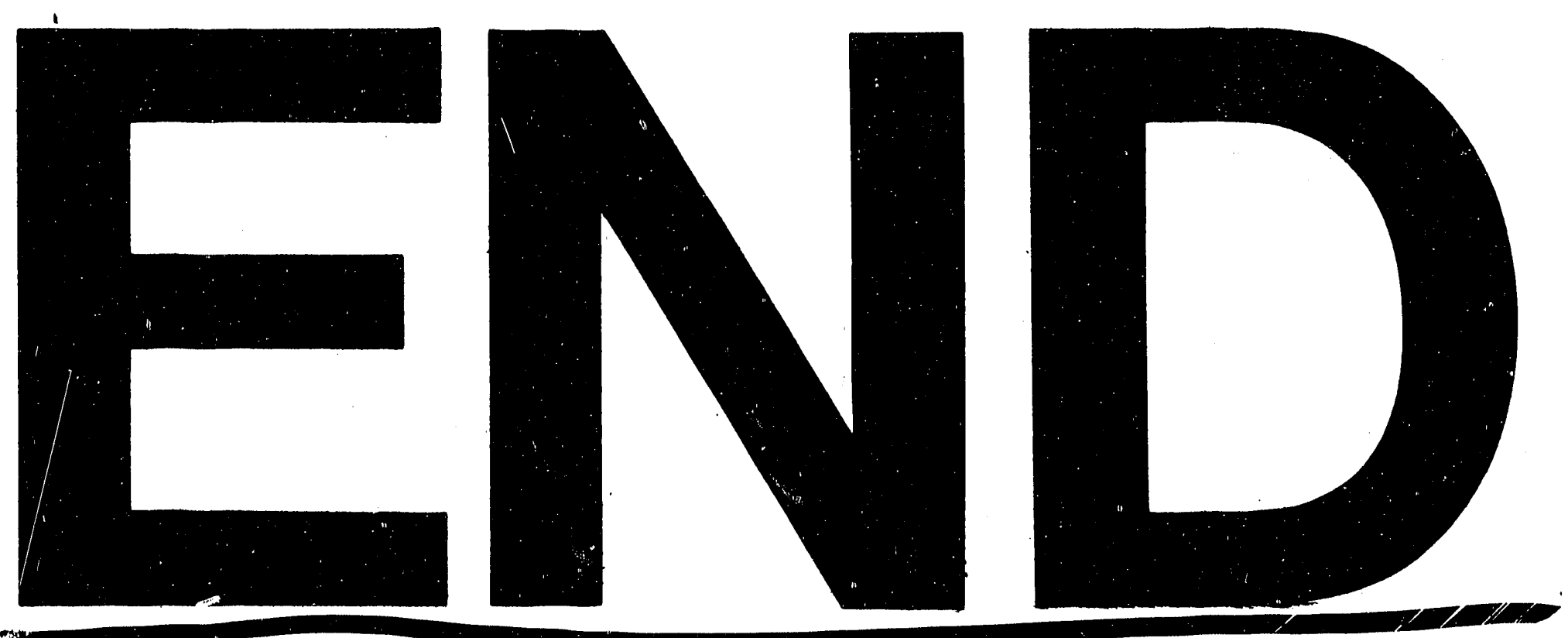

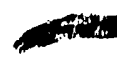

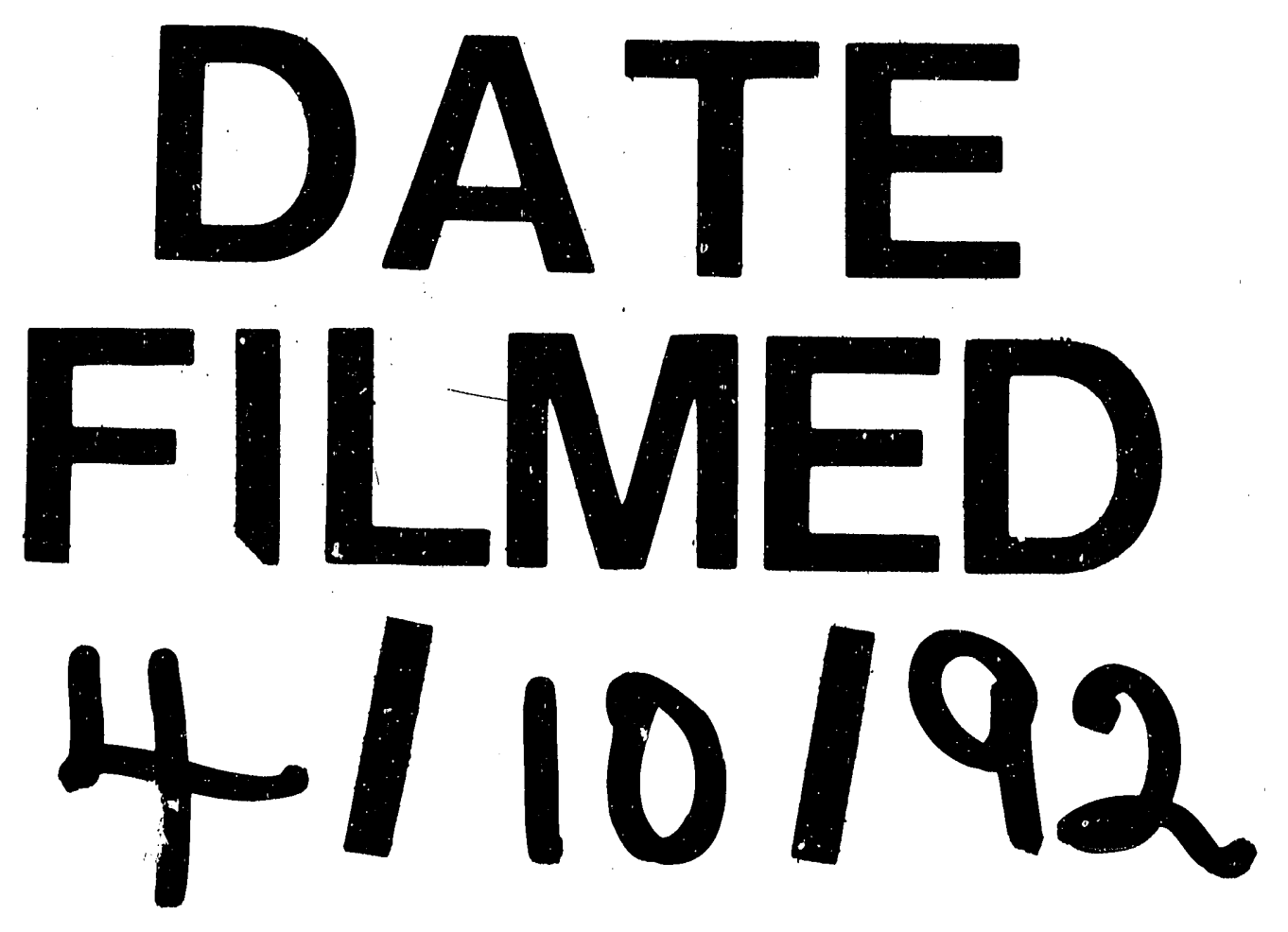


